\title{
Cerebral venous sinus thrombosis in a patient with delirium secondary to COVID-19
}

\author{
Madeleine Stephen MD, Laila Alshafai MBBS, Allan Detsky MD PhD, Michael Fralick MD PhD
}

Cite as: CMAJ 2022 January 10;194:E15-6. doi: 10.1503/cmaj.211745

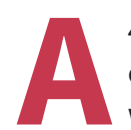

46-year-old woman was transferred to an internal medicine ward after a 5-week stay in the intensive care unit, where she was treated for severe acute respiratory distress syndrome secondary to COVID-19. She had no past medical history and had not received a SARS-CoV-2 vaccine.

Her predominant symptoms while on the ward included confusion, fear, paranoia and a fluctuating ability to speak. Our initial diagnosis was delirium secondary to critical illness and prolonged exposure to sedating medications. After a week on the ward, the symptoms persisted, with no improvement. A noncontrast computed tomography (CT) scan of the patient's head identified a small focal high density in the right lateral transverse sinus into the junction of the right sigmoid sinus, which raised concern for an acute dural venous thrombosis (Figure 1A). A CT venogram showed extensive thromboses extending into the right jugular bulb and right internal jugular vein (Figure 1B). We started the patient on therapeutic anticoagulation with lowmolecular-weight heparin (planned duration 6 months), and her delirium resolved 5 days later. Four months later, she made a full recovery and was speaking normally.

Our case is an important reminder that cerebral venous sinus thrombosis (CVST) is associated with COVID-19 as well as the AstraZeneca ChAdOx1-S SARS-CoV-2 vaccine. ${ }^{1,2}$ A report from Singapore estimated a $0.005 \%$ incidence of CVST in people positive for SARS-CoV-2. ${ }^{3}$ A systematic review found that, among patients with concomitant COVID-19 and CVST, the most common symptom was altered mental status (39\%), 75\% of patients had CVST identified in the transverse sinus on imaging, and the mortality rate was about $40 \% .^{3}$ The diagnosis of CVST is often missed because symptoms are nonspecific and often subacute. ${ }^{3,4}$ Guidelines suggest that CVST be considered in patients with focal neurologic deficits with accompanying headache and either seizure or altered mental status. ${ }^{3,4}$ The recommended treatment for CVST is low-molecular-weight heparin for 3-6 months. ${ }^{5}$

\section{References}

1. Franchini M, Liumbruno GM, Pezzo M. COVID-19 vaccine-associated immune thrombosis and thrombocytopenia (VITT): diagnostic and therapeutic recommendations for a new syndrome. Eur J Haematol 2021;107:173-80.

2. Murk W, Gierada M, Fralick M, et al. Diagnosis-wide analysis of COVID-19 complications: an exposure-crossover study. CMAJ 2021;193:E10-8.

3. Tu TM, Goh C, Tan YK, et al. Cerebral venous thrombosis in patients with
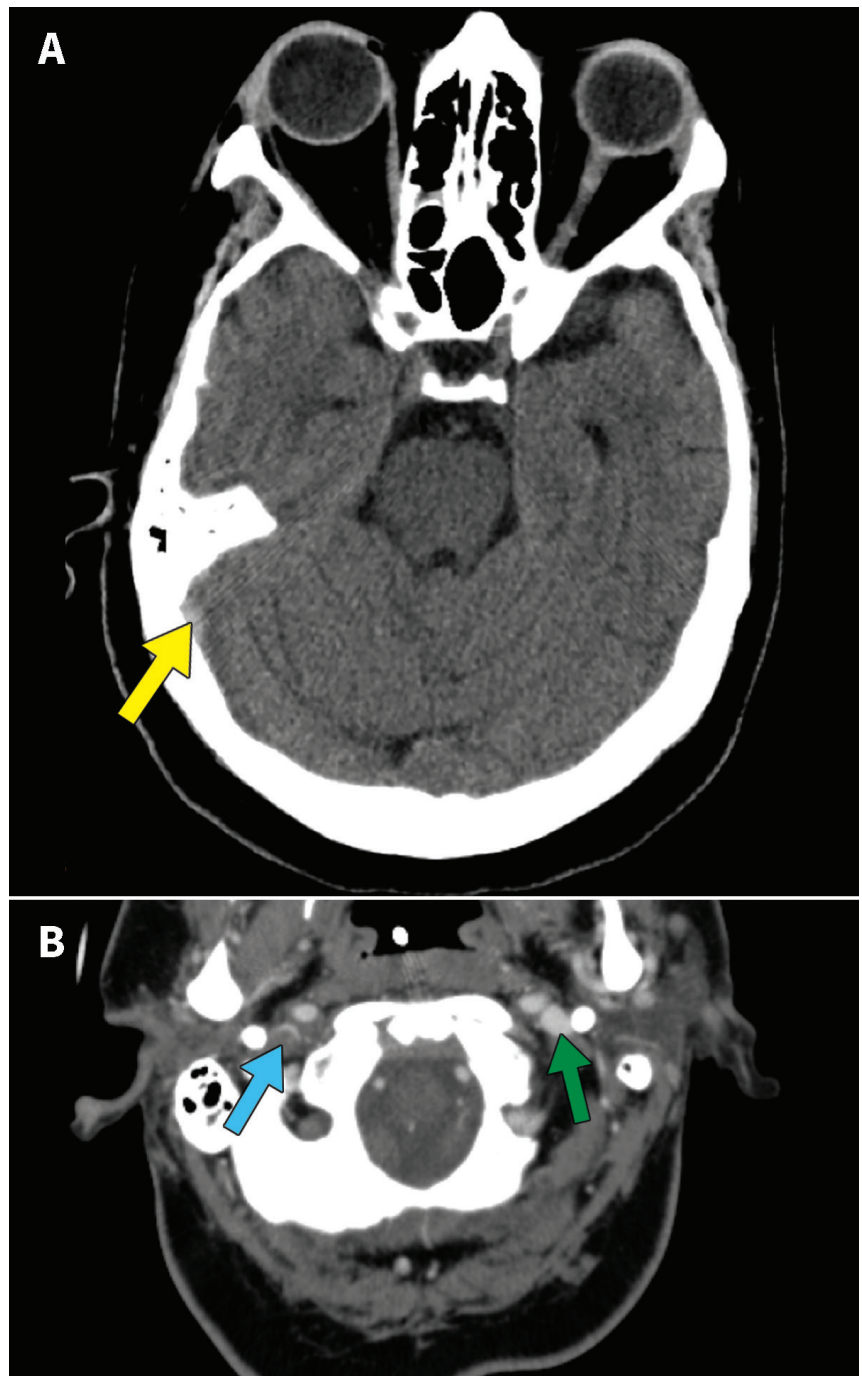

Figure: 1: Computed tomography (CT) scans of the head of a 46-year-old woman with cerebral venous sinus thrombosis. (A) Unenhanced CT (axial slice), showing a subtle focal high density in the right lateral transverse sinus into the junction with the right transverse sinus (arrow) and in the right sigmoid sinus (not shown), raising concern for acute dural venous thrombosis. There is no associated edema or hemorrhage. (B) CT venogram, showing near occlusive thrombosis in the right internal jugular vein (dark grey) just below the level of the skull base (blue arrow). Extensive acute thrombosis extended to the right sigmoid sinus and jugular bulb (not shown). Contrast is seen on the contralateral side (light grey), indicating patency (green arrow). 
COVID-19 infection: a case series and systematic review. J Stroke Cerebrovasc Dis 2020;29:105379.

4. Einhäupl K, Stam J, Bousser MG, et al. EFNS guideline on the treatment of cerebral venous and sinus thrombosis in adult patients. Eur J Neurol 2010;17:1229-35.

5. Idiculla PS, Gurala D, Palanisamy M, et al. Cerebral venous thrombosis: a comprehensive review. Eur Neurol 2020;83:369-79.

Competing interests: Michael Fralick is a consultant for a start-up company, Proof Diagnostics, that has created a point-of-care testing device for SARS-CoV-2. He also holds grants from the Canadian Institutes of Health Research and the Canadian military for clinical trials to identify treatments for COVID-19, outside the submitted work. No other competing interests were declared.

This article has been peer reviewed.

The authors have obtained patient consent.

Affiliations: Department of Medicine (Stephen, Detsky, Fralick) and of Radiology (Alshafai), Sinai Health System; Institute of Health Policy Management and Evaluation (Detsky); Department of Medicine (Detsky), University of Toronto, Toronto, Ont.

Content licence: This is an Open Access article distributed in accordance with the terms of the Creative Commons Attribution (CC BY-NCND 4.0) licence, which permits use, distribution and reproduction in any medium, provided that the original publication is properly cited, the use is noncommercial (i.e., research or educational use), and no modifications or adaptations are made. See: https://creativecommons.org/ licenses/by-nc-nd/4.0/

Correspondence to: Michael Fralick, mike.fralick@mail.utoronto.ca

Clinical images are chosen because they are particularly intriguing, classic or dramatic. Submissions of clear, appropriately labelled highresolution images must be accompanied by a figure caption. A brief explanation (300 words maximum) of the educational importance of the images with minimal references is required. The patient's written consent for publication must be obtained before submission. 